\title{
Shakhbazian compact groups: Poor clusters of galaxies
}

\author{
H. M. Tovmassian ${ }^{1}$ and H. Tiersch ${ }^{2}$ \\ 1 Instituto Nacional de Astrofísica Optica y Electrónica, AP 51 y 216, 72000 Puebla, Pue., México \\ e-mail: hrant@inaoep.mx \\ 2 Sternwarte Königsleiten, München, Germany \\ e-mail: htiersch@uni.de
}

Received 3 May 2001 / Accepted 26 July 2001

\begin{abstract}
The environment of 105 elongated $(b / a<0.5)$ ShCGs is investigated for galaxies probably associated with them. Counts of galaxies are made in two reciprocally orthogonal stripes of $5^{\prime}$ width and $20^{\prime}$ length passing through the center of each elongated group. The orientation of the first stripe is determined by the orientation of elongation of the distribution of the principal members of groups. It is found that there is an excess of galaxies in the narrow stripes oriented along the elongation of the group. Such peculiar distribution of the loose group members allows us to conclude that they are gravitationally bound with the compact group, and rotate around the common gravitational center. It is shown that the new-found distant members compose, together with the principal members of the compact group, a gravitationally bound, and, most probably, virialized system. All members of the system rotate around the common gravitational center. It is concluded that ShCGs are the cores of poor clusters of galaxies. They must be not young, and are relatively stable configurations. The number of such groups could be very high in the Universe.
\end{abstract}

Key words. galaxies: general, abundances, clusters: general

\section{Introduction}

The problem of formation, existence and evolution of compact groups (CGs) of galaxies is a very intriguing one. $N$-body simulations showed that due to the proximity of member galaxies of CGs to each other they start to merge after a small number of crossing times (Barnes 1985, 1989; Mamon 1986). Each group should finally merge to one elliptical galaxy on a time scale of $\sim 10^{9}$ years. On the other hand, it has been shown that CGs are located in a dense environment of galaxies (Rose 1977; Sulentic 1987). The presence of loose groups (LGs) around Hickson compact groups (HCGs) has been stated by many others (e.g. Rood \& Williams 1989; Ramella et al. 1994; Ribeiro et al. 1998; Barton et al. 1998; Coziol et al. 1998; Vennik et al. 1993). Mamon (1986) and Walke \& Mamon (1989) suggested that CGs are not real physical entities, but are chance alignments of galaxies in LGs. According to Hernquist et al. (1995) and Ostriker et al. (1995) HCGs are filaments seen end-on. Much evidence has been presented, however, for the reality of HCGs (Hickson \& Rood 1988; Mendes de Oliveira \& Giraud 1994; Mendes de Oliveira 1995; Ponman et al. 1996; Hickson 1997; Tovmassian et al. 1998). Diaferio et al. (1994, 1995), Governato et al. (1996) suggested that field galaxies from relatively denser

Send offprint requests to: H. M. Tovmassian, e-mail: hrant@inaoep.mx environments fall from time to time onto HCGs, and thus maintain their existence.

Tovmassian et al. (2001) showed that galaxies of LGs have been found by Rood \& Williams (1989) and Ramella et al. (1994) mostly in the neighborhood of those HCGs the direction of elongation of which is oriented at about $45^{\circ}$ to the line of sight. Moreover, Tovmassian (2001) showed that LGs in the environment of HCGs may be found more efficiently, if one looks for LG members in areas located along the elongation of the corresponding HCG. It means that members of LGs are distributed along the same direction as the principal members of HCGs. Tovmassian \& Chavushyan (2000) found that the radial velocity dispersion (RVD) of the LG members depends on the elongation of the group. Such peculiar distribution of the LG members in narrow stripes oriented along the direction in which principal members of the CG are distributed, and the dependence of the RVD on the elongation of the group shows that the LG members are, apparently, physically associated with the CG, and possibly rotate around the common gravitational center (Tovmassian \& Chavushyan 2000). Tovmassian \& Chavushyan (2000) concluded that HCGs are, thus, more stable configurations than it has been assumed before. Governato et al. (1991) and Zepf \& Whitmore (1991) showed earlier that if to assume that most massive galaxies of CGs are even in quasi stationary orbits, the actual merging time of the 
group can be considerably delayed. If to take into account that these orbits have also regular orientation along the group (Tovmassian et al. 1998; Tovmassian 2001), the life time of groups may apparently be higher.

In this paper we searched the environments of Shakhbazian compact groups (ShCGs) for faint companions. Ten lists of ShCGs have been compiled during 1973-1979 by Shakhbazian, Petrosian, Baier and Tiersch (Shakhbazian 1973; Shakhbazian et al. 1974; Petrosian 1974, 1978; Baier et al. 1974; Baier \& Tiersch 1975, 1976a, 1976b, 1978, 1979). The total ShCG survey contains 377 groups. It is the largest known sample of CGs. ShCGs are more member-rich than HCGs. Each group contains 5-15 members with apparent magnitudes of individual galaxies in the range of $14^{\mathrm{m}}-19^{\mathrm{m}}$. The distances between galaxies are typically only $3-5$ times the diameter of galaxies. ShCGs are, on average, at least three times further than HCGs (Tovmassian et al. 1998). Therefore, only relatively bright members of LGs, if they exist, could be detected, and, hence, the number of found galaxies may be small.

\section{Counts of galaxies}

The study of HCGs showed that faint galaxies in their environments are distributed along the direction of the elongation determined by the principal members of the corresponding CG (Tovmassian \& Chavushyan 2000; Tovmassian et al. 2001; Tovmassian 2001). Therefore, sufficiently elongated ShCGs with $b / a<0.5^{1}$ were selected to look for galaxies in their environment.

Among the total list of 377 ShCGs there are 179 groups with $b / a<0.5$. ShCGs have been selected as compact groups of compact galaxies, as the latter appeared on the Palomar Prints. Spectral observations showed later that some groups, e.g. ShCG 12, 78, 146, etc. (Tovmassian et al. 1998) consist mainly of stars. Such false groups would mask the searched peculiar distribution of the LG galaxies around CGs. For this reason we inspected on the DSS the images of galaxies in those groups in which almost no galaxies were identified in the APS catalog ${ }^{2}$. Revision of images of members of ShCGs with the use of the APS and DSS showed that many members of the groups ShCG 12, 25, 28, 99, 106, 107, 108, 115, 116, 119, 124, 140, 146, 148, $232,239,249,250,274,287,290,293,305,316$, and 319 are most likely stars. These groups were considered as, probably, false ones, and were excluded from further study. The $b / a$ ratio for some groups (ShCG 52, 83, 104, 248) became higher than 0.5 after exclusion of starlike members which turned out to be stars. These groups were also excluded

\footnotetext{
1 The parameter $b / a$, where $a$ is the angular distance between the most widely separated galaxies in the group, and $b$ is the sum of the angular distances $b_{1}$ and $b_{2}$ of the most distant galaxies, on either side of the line $a$ joining the most separated galaxies (Rood 1979), characterizes the elongation of the group.

2 The APS databases are supported by the National Aeronautics and Space Administration and the University of Minnesota, and are available at http://aps.umn.edu/
}

from the study. Nine groups (ShCG 6, 38, 54, 120, 165, $190,233,361$, and 362) located within the known Abell or Zwicky clusters of galaxies, were excluded as well. After exclusion of the mentioned ShCGs 142 groups were left. The APS catalog was used for counts. The counts were made in 105 of those ShCGs for which the APS data were available as of 1 December 2000.

We counted galaxies in two orthogonal narrow stripes passing through the center of each group (Fig. 1). The first stripe was set to be oriented in the direction of the elongation outlined by principal members of the corresponding ShCG. The position angle of this direction is determined with an accuracy of a few degrees. Since the largest dimension of the majority of ShCGs is about $2^{\prime}-3^{\prime}$, a value of $5^{\prime}$ was chosen as the width of the searched stripes. The length of stripes is $20^{\prime}$. At a mean redshift $z \sim 0.1$ of ShCGs (Tovmassian et al. 1998) the width and length of stripes correspond, on average, to $\sim 600 \mathrm{kpc}$ and $\sim 2.3 \mathrm{Mpc}$, respectively ${ }^{3}$.

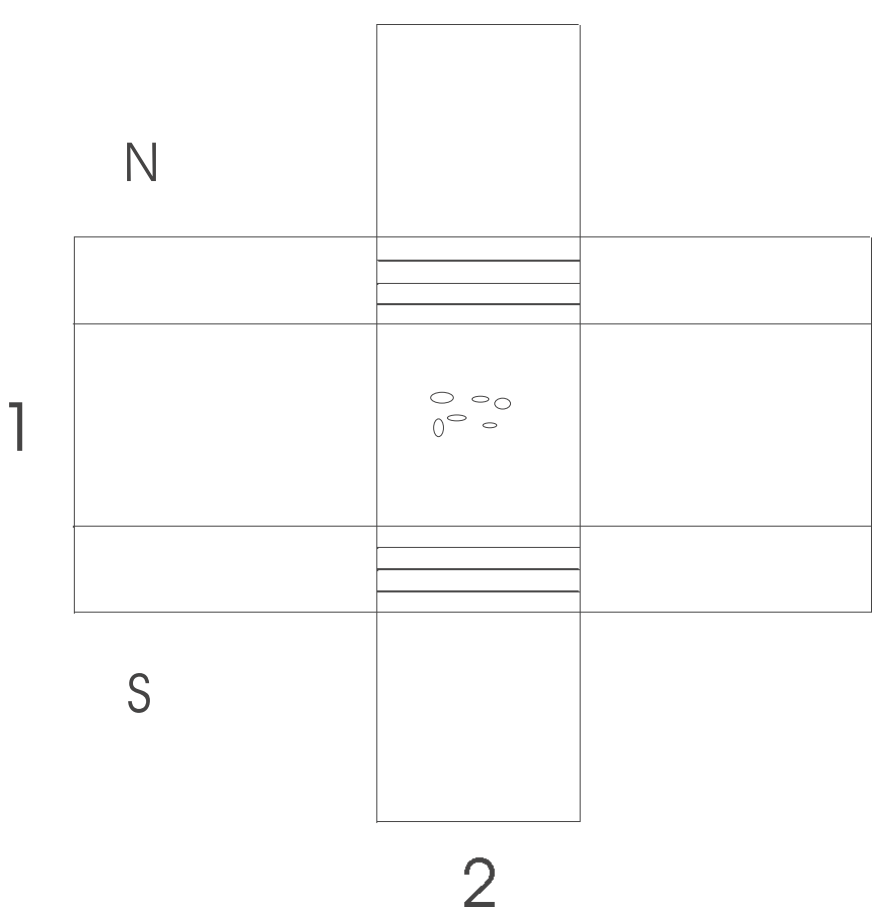

Fig. 1. The scheme of stripes where counts were made.

The limiting magnitude of the counted galaxies is $22^{\mathrm{m}}$ in $R$. The photometric accuracy of the APS may be worse than the claimed value of about 0.25 . This may introduce an uncertainty at the limit of the APS detection. One has also to take into account that there could be some selection effects related to misclassification of galaxies and stars in the APS. Indeed, two nearby stars on the sky or a fuzzy image of a star could be recognized as a galaxy. On the other hand, a galaxy with a bright core may be misclassified as a star. Such misidentification may have some influence on the counts made. However, as is seen

\footnotetext{
${ }^{3} H=75 \mathrm{~km} \mathrm{~s}^{-1} \mathrm{Mpc}^{-1}$ throughout this paper.
} 
below, such selection effects do not have any significant influence in the case of the counts made.

The numbers, $N_{\mathrm{c}}$, of galaxies in the first stripe, oriented along the elongation of the corresponding group, and the numbers, $N_{\mathrm{o}}$, in the orthogonal stripe were counted. Since the aim of the counts made was to see if there is a systematic difference in the numbers of galaxies in two reciprocally orthogonal stripes, the counts do not include galaxies in the central square with a $5^{\prime}$ side. Hence, counts were made in stripes with areas $5^{\prime} \times 15^{\prime}=75 \square^{\prime}$. The identified members of ShCGs which were out of the common central square, were not included in the counts. Then the difference, $\Delta_{\mathrm{c}}$ between $N_{\mathrm{c}}$ and $N_{\mathrm{o}}$ was calculated for each group.

For comparison, additional counts were made also in two half as narrow stripes on both sides (to the north, and to the south) of the first stripe (the area of each of these narrow stripes is $2.5^{\prime} \times 20^{\prime}=50 \square^{\prime}$ ). Then the differences $\Delta_{\mathrm{n}-\mathrm{s}}=N_{\mathrm{n}}-N_{\mathrm{s}}$ between the numbers of galaxies in the northern and southern stripes were calculated. The differences $\Delta_{\mathrm{s}}$ between the numbers of galaxies $N_{\mathrm{n}+\mathrm{s}}=N_{\mathrm{n}}+N_{\mathrm{s}}$ in two side stripes and $N_{\mathrm{o}}$ in the orthogonal stripe were calculated as well. In the latter case, to make equal the sum area of two side stripes to that of the central stripe $\left(\left(2.5^{\prime}+2.5^{\prime}\right) \times 15^{\prime}\right)$, the counts were not made in those parts of the side stripes (shaded in Fig. 1) which overlap with the orthogonal stripe.

The results of counts are presented in Table 1. In the first column the designation of the group is given. The right ascension and declination of the center of the searched stripe are presented in Cols. 2 and 3. The center of the searched area mostly coincides with that given in the original ShCG lists (Shakhbazian 1973; Shakhbazian et al. 1974; Petrosian 1974; Petrosian 1978; Baier et al. 1974; Baier \& Tiersch 1975, 1976a, 1976b, 1978, 1979). The position angle of the first stripe is given in Col. 4. The numbers $N_{\mathrm{c}}$ and $N_{\mathrm{o}}$ of galaxies in the central and orthogonal stripes are given in Cols. 5 and 6 respectively. The difference $\Delta_{c}$ is presented in Col. 7. The differences $\Delta_{\mathrm{n}-\mathrm{s}}$ and $\Delta_{\mathrm{s}}$ are presented in Cols. 8 and 9 , respectively.

\section{Discussion}

If the distribution of galaxies in the environment of ShCGs does not depend on the orientation of the elongation of the latter, then all three considered differences $\Delta_{\mathrm{c}}, \Delta_{\mathrm{n}-\mathrm{s}}$, and $\Delta_{\mathrm{s}}$ would apparently be determined by noise, and would have a normal distribution around zero. The histogram of the distribution of differences $\Delta_{c}$ between numbers of galaxies in the central stripe oriented along the elongation of the group and in the orthogonal one, is presented on the upper frame of Fig. 2. On the same figure the differences $\Delta_{n-s}$ between numbers of galaxies in two side stripes (middle frame), and differences $\Delta_{\mathrm{S}}$ between the sum of the numbers of galaxies in two side stripes and in the central orthogonal stripe (lower frame) are presented. The results of counts are summarized in Table 2. The KolmogorovSmirnov test showed that with a probability $P=99 \%$ the distributions of all three considered differences $\Delta_{\mathrm{c}}, \Delta_{\mathrm{n}-\mathrm{s}}$ and $\Delta_{\mathrm{S}}$ are normal. Mean values of the last two differences are, as it was expected, close to zero: $1.0 \pm 6.6$ and $-0.2 \pm 7.5$ respectively. Meanwhile, the mean value of differences $\Delta_{c}$ is $3.7 \pm 6.9$.

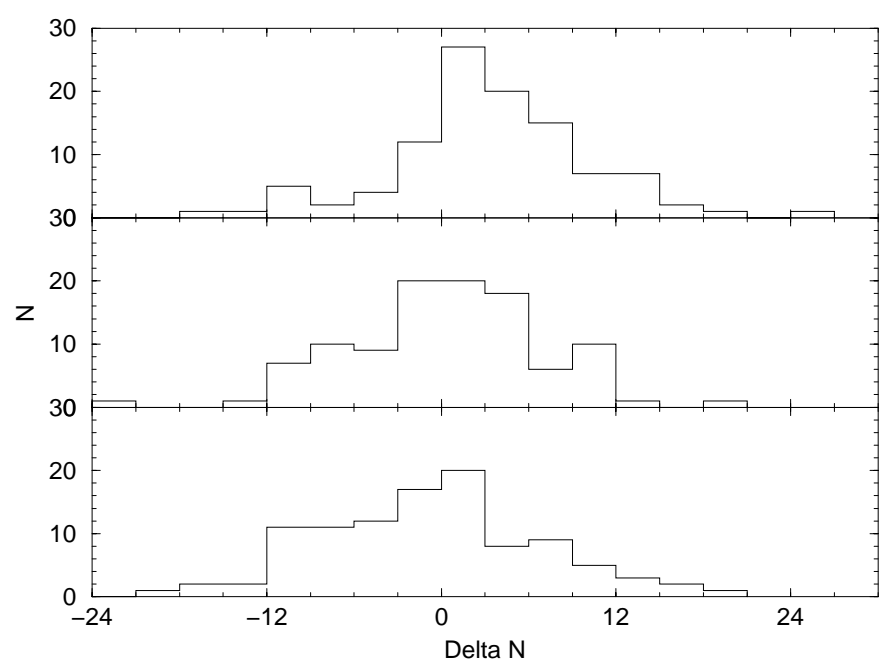

Fig. 2. The hystograms of distribution of differences $\Delta_{\mathrm{c}}$ (up), $\Delta_{\mathrm{n}-\mathrm{s}}\left(\right.$ middle), and $\Delta_{\mathrm{s}}$ (lower).

Considering the differences $\Delta_{\mathrm{n}-\mathrm{s}}$ and $\Delta_{\mathrm{s}}$ as a control samples, we may assume that a LG is, probably, detected, if $\Delta_{\mathrm{c}}$ exceeds the dispersion $\sim 8$ of both these distributions. It is prominent that $\Delta_{\mathrm{c}} \geq 8$ in the case of 26 ShCGs, while the number of groups with $\Delta_{\mathrm{c}} \leq-8$ is only 8 . In the samples of differences $\Delta_{\mathrm{n}-\mathrm{s}}$ and $\Delta_{\mathrm{s}}$ the numbers of groups with large positive values $(\geq 8)$ of $\Delta_{\mathrm{n}-\mathrm{s}}$ and $\Delta_{\mathrm{s}}$ are smaller: 15 and 19 respectively. These numbers differ very little from the numbers of groups with large negative $(\leq-8)$ values of $\Delta_{\mathrm{n}-\mathrm{s}}$ 's and $\Delta_{\mathrm{s}}$ 's: 10 and 18 respectively. Hence, it is very probable that most of the 26 ShCGs with $\Delta_{\mathrm{c}} \geq$ 8 are associated with LGs. There are, on average, $12 \pm$ 4 galaxies in the environment of each of these 26 ShCGs.

The two-sample Mann-Whitney rank sum test showed that the hypothesis that the distribution of $\Delta_{c}$ is of the same parent distribution as that of $\Delta_{\mathrm{n}-\mathrm{s}}$ and that of $\Delta_{\mathrm{s}}$, is rejected with probabilities $P>99.8 \%$ and $P>99.99 \%$, respectively. Hence, an excess of, on average, four galaxies is found in the environment of the studied elongated ShCGs. And, as in the case of HCGs (Tovmassian 2001), these galaxies are located along the direction in which the principal members of CGs are distributed.

The two-sample Mann-Whitney rank sum test showed that the distribution of differences $\Delta_{\mathrm{S}}$ between the sum of the numbers of galaxies in two side stripes and in the central orthogonal stripe does not differ from the distribution of $\Delta_{\mathrm{n}-\mathrm{s}}$ with a significance level $P=10^{-8}$. It means that members of LG galaxies are found merely in the narrow central stripe with $5^{\prime}$ width. It is not excluded that some amount of LG members may be located in the central square in the immediate vicinities of bright members of groups. 
Table 1. The results of counts.

\begin{tabular}{|c|c|c|c|c|c|c|c|c|}
\hline ShCG & $\begin{array}{r}\text { RA } \\
2000.0\end{array}$ & $\begin{array}{r}\text { Dec } \\
2000.0\end{array}$ & $\begin{array}{l}\theta \\
\circ\end{array}$ & $N_{\mathrm{c}}$ & $N_{\mathrm{o}}$ & $\Delta_{\mathrm{c}}$ & $\Delta_{\mathrm{n}-\mathrm{s}}$ & $\Delta_{\mathrm{s}}$ \\
\hline 3 & 111516.0 & 534040 & 117 & 38 & 25 & +13 & +10 & +8 \\
\hline 4 & 161945.0 & 614230 & 95 & 20 & 11 & +9 & +6 & -3 \\
\hline 9 & 132405.3 & 190148 & 28 & 33 & 25 & +8 & +3 & +3 \\
\hline 10 & 141048.0 & 461540 & 89 & 33 & 24 & +9 & +3 & +2 \\
\hline 14 & 142520.0 & 471501 & 33 & 25 & 17 & +8 & +4 & +9 \\
\hline 24 & 234656.6 & -005302 & 89 & 38 & 35 & +3 & +3 & -10 \\
\hline 27 & 233242.3 & 013300 & 151 & 18 & 27 & -9 & +1 & -8 \\
\hline 31 & 005817.4 & 135445 & 109 & 24 & 24 & 0 & -4 & -4 \\
\hline 32 & $\begin{array}{lll}01 & 01 & 47.1\end{array}$ & -013450 & 172 & 23 & 13 & +10 & -1 & -2 \\
\hline 33 & 010342.6 & -010900 & 18 & 13 & 13 & 0 & +4 & -2 \\
\hline 41 & 012901.5 & 074027 & 37 & 24 & 22 & +2 & +6 & -10 \\
\hline 43 & 013827.1 & 083108 & 54 & 39 & 39 & 0 & +5 & -15 \\
\hline 44 & 014052.9 & 025113 & 166 & 22 & 24 & -2 & +1 & -2 \\
\hline 46 & 020512.4 & 032058 & 25 & 23 & 11 & +12 & -3 & +8 \\
\hline 47 & 020600.6 & 025245 & 124 & 20 & 9 & +11 & +2 & +10 \\
\hline 48 & 094807.5 & 465232 & 99 & 27 & 21 & +6 & -2 & +1 \\
\hline 49 & 101519.0 & 385535 & 30 & 40 & 29 & +11 & +1 & +1 \\
\hline 51 & 103032.9 & 391257 & 94 & 28 & 26 & +2 & -4 & -6 \\
\hline 55 & 104334.5 & 482154 & 105 & 22 & 21 & +1 & +4 & +2 \\
\hline 56 & 104330.0 & 420118 & 54 & 26 & 25 & +1 & -9 & -1 \\
\hline 57 & 104520.4 & 493200 & 30 & 27 & 38 & -11 & +3 & -12 \\
\hline 68 & 114738.8 & 391616 & 10 & 10 & 7 & +3 & +5 & +2 \\
\hline 69 & 115214.4 & 390647 & 5 & 23 & 17 & +6 & +4 & 0 \\
\hline 74 & 142108.4 & 420355 & 105 & 29 & 23 & +6 & -2 & +2 \\
\hline 75 & 142736.1 & 384600 & 135 & 23 & 15 & +8 & +3 & 0 \\
\hline 79 & 153239.8 & 430400 & 40 & 22 & 15 & +7 & +5 & +3 \\
\hline 81 & 215758.0 & -014534 & 48 & 41 & 35 & +6 & +11 & +13 \\
\hline 84 & 233437.5 & 075925 & 157 & 8 & 9 & -1 & -5 & 0 \\
\hline 95 & 082837.9 & 501743 & 97 & 14 & 10 & +4 & -6 & +12 \\
\hline 121 & 113052.4 & 375312 & 72 & 11 & 8 & +3 & +6 & +8 \\
\hline 122 & 114313.6 & 571820 & 93 & 23 & 19 & +4 & -2 & -3 \\
\hline 131 & 143805.6 & 624403 & 77 & 8 & 4 & +4 & 0 & 0 \\
\hline 141 & 010415.7 & -013312 & 104 & 15 & 8 & +7 & -2 & -4 \\
\hline 142 & $\begin{array}{lll}01 & 471.1\end{array}$ & 032523 & 101 & 32 & 19 & +13 & +6 & -5 \\
\hline 145 & $\begin{array}{lll}02 & 16 & 36.7\end{array}$ & -020830 & 34 & 31 & 29 & +2 & -3 & -8 \\
\hline 182 & $\begin{array}{lll}08 & 38 & 19.1\end{array}$ & 294454 & 110 & 26 & 21 & +5 & -5 & +1 \\
\hline 183 & 084434.0 & 271710 & 26 & 23 & 19 & +4 & +6 & -11 \\
\hline 184 & $\begin{array}{lll}09 & 08 & 07.9\end{array}$ & 303628 & 55 & 16 & 12 & +4 & 0 & +8 \\
\hline 185 & 092226.0 & 313241 & 44 & 23 & 10 & +13 & +7 & +8 \\
\hline 186 & 092252.5 & 285524 & 26 & 28 & 24 & +4 & +10 & -2 \\
\hline 187 & 094027.0 & 305903 & 49 & 13 & 12 & +1 & +4 & +4 \\
\hline 189 & 102755.3 & 321543 & 12 & 30 & 26 & +4 & -7 & -9 \\
\hline 195 & 110433.2 & 284225 & 139 & 14 & 18 & -4 & +9 & +2 \\
\hline 196 & 110546.4 & 282430 & 48 & 23 & 18 & +5 & -4 & -5 \\
\hline 200 & 114113.8 & 272610 & 10 & 16 & 14 & +2 & +10 & +2 \\
\hline 204 & 123007.6 & 273506 & 43 & 32 & 33 & -1 & -10 & -1 \\
\hline 209 & 131039.2 & 314416 & 100 & 36 & 23 & +13 & +12 & +16 \\
\hline 211 & 133630.0 & 283434 & 174 & 20 & 26 & -6 & -7 & -8 \\
\hline 213 & 134511.6 & 265331 & 177 & 43 & 26 & +17 & +7 & +3 \\
\hline 214 & 134523.7 & 320636 & 53 & 36 & 15 & +21 & +5 & +15 \\
\hline 220 & 155930.0 & 294216 & 82 & 21 & 22 & -1 & +11 & +4 \\
\hline 221 & 153655.0 & 304124 & 98 & 44 & 18 & +26 & +7 & +11 \\
\hline 222 & 154338.7 & 313312 & 95 & 38 & 37 & +1 & -1 & -11 \\
\hline
\end{tabular}


Table 1. continued.

\begin{tabular}{|c|c|c|c|c|c|c|c|c|}
\hline ShCG & $\begin{array}{r}\mathrm{RA} \\
2000.0\end{array}$ & $\begin{array}{r}\text { Dec } \\
2000.0\end{array}$ & $\begin{array}{l}\theta \\
0\end{array}$ & $N_{\mathrm{c}}$ & $N_{\mathrm{o}}$ & $\Delta_{\mathrm{c}}$ & $\Delta_{\mathrm{n}-\mathrm{s}}$ & $\Delta_{\mathrm{s}}$ \\
\hline 230 & 095136.0 & 320851 & 53 & 25 & 11 & +14 & +10 & +1 \\
\hline 231 & 100141.4 & 381845 & 23 & 24 & 35 & -11 & +10 & -14 \\
\hline 235 & 105620.0 & 321800 & 89 & 31 & 24 & +7 & -1 & +2 \\
\hline 237 & 110528.6 & 380054 & 74 & 24 & 16 & +8 & +5 & +5 \\
\hline 240 & 113340.0 & 360352 & 127 & 18 & 27 & -9 & +15 & +5 \\
\hline 244 & 121705.9 & 351800 & 131 & 18 & 16 & +2 & +12 & -4 \\
\hline 246 & 123822.7 & 321306 & 65 & 37 & 31 & +6 & +1 & -4 \\
\hline 251 & 133653.6 & 364933 & 41 & 22 & 16 & +6 & 0 & -2 \\
\hline 255 & 141042.0 & 321542 & 125 & 37 & 35 & +2 & -1 & 0 \\
\hline 257 & 144656.8 & 373404 & 150 & 28 & 27 & +1 & -9 & +4 \\
\hline 259 & 153930.7 & 375044 & 45 & 18 & 26 & -8 & -10 & -6 \\
\hline 260 & 154224.2 & 345400 & 92 & 28 & 27 & +1 & +8 & +17 \\
\hline 272 & 023210.5 & -131246 & 6 & 15 & 13 & +2 & +10 & +7 \\
\hline 273 & 025236.0 & -130600 & 144 & 9 & 11 & -2 & +1 & -4 \\
\hline 276 & 031714.0 & -135125 & 32 & 25 & 22 & +3 & -1 & -4 \\
\hline 279 & 041254.3 & -082904 & 131 & 32 & 25 & +7 & +6 & +10 \\
\hline 289 & 135809.3 & -125300 & 95 & 36 & 35 & +1 & +2 & -7 \\
\hline 291 & 140618.2 & -102857 & 113 & 35 & 34 & +1 & +19 & -3 \\
\hline 295 & 150559.4 & -122451 & 11 & 46 & 39 & +7 & -4 & +1 \\
\hline 297 & 214217.5 & -115328 & 138 & 42 & 34 & +8 & -8 & -6 \\
\hline 299 & 221815.2 & -133933 & 60 & 31 & 35 & -4 & +2 & -2 \\
\hline 300 & 222431.2 & -112058 & 88 & 23 & 19 & +4 & -9 & +11 \\
\hline 304 & 234340.0 & -122835 & 91 & 19 & 20 & -1 & +2 & +1 \\
\hline 308 & 003711.7 & -074154 & 161 & 23 & 16 & +7 & -1 & +20 \\
\hline 309 & 005116.8 & -072433 & 2 & 12 & 24 & -12 & -3 & -3 \\
\hline 314 & 012545.7 & -032944 & 107 & 28 & 12 & +16 & +6 & +6 \\
\hline 317 & 021041.8 & -063512 & 130 & 31 & 19 & +12 & +1 & +1 \\
\hline 318 & 110936.9 & -042020 & 92 & 11 & 12 & -1 & +5 & -1 \\
\hline 320 & 111446.7 & -062140 & 22 & 11 & 10 & +1 & 0 & -1 \\
\hline 321 & 111559.1 & -035602 & 145 & 20 & 14 & +6 & +11 & -2 \\
\hline 322 & 112341.7 & -041302 & 93 & 24 & 21 & +3 & +2 & -1 \\
\hline 323 & 121917.3 & -072414 & 123 & 41 & 39 & +2 & -6 & -9 \\
\hline 324 & 122544.7 & -083138 & 90 & 20 & 14 & +6 & +1 & -5 \\
\hline 325 & 124348.7 & -064734 & 50 & 25 & 23 & +2 & -3 & -10 \\
\hline 331 & 222527.1 & -024700 & 13 & 14 & 31 & -17 & 0 & -15 \\
\hline 332 & 225212.8 & -050007 & 124 & 29 & 31 & -2 & -2 & -11 \\
\hline 333 & 220203.4 & -054300 & 152 & 25 & 23 & +2 & -1 & +6 \\
\hline 334 & 230652.2 & -043332 & 159 & 24 & 33 & -11 & +4 & -19 \\
\hline 338 & 000423.3 & 034918 & 27 & 20 & 25 & -5 & +3 & -3 \\
\hline 343 & 084113.1 & 073201 & 42 & 26 & 24 & +2 & -10 & +14 \\
\hline 344 & 084732.3 & 034153 & 155 & 37 & 27 & +10 & -9 & -6 \\
\hline 347 & 091732.9 & 074230 & 41 & 31 & 27 & +4 & +1 & +8 \\
\hline 349 & 100635.5 & 065548 & 120 & 31 & 34 & -3 & -14 & -2 \\
\hline 350 & 110001.2 & 082422 & 131 & 21 & 19 & +2 & -7 & -10 \\
\hline 355 & 131211.6 & 071829 & 95 & 22 & 17 & +5 & 0 & +3 \\
\hline 356 & 133039.3 & 112409 & 41 & 46 & 36 & +10 & +1 & +8 \\
\hline 367 & 090144.0 & 214207 & 86 & 22 & 23 & -1 & -1 & -11 \\
\hline 370 & 095020.9 & 231638 & 11 & 34 & 19 & +15 & -4 & +5 \\
\hline 371 & 114333.1 & 215348 & 37 & 40 & 33 & +7 & -2 & +2 \\
\hline 374 & 131559.1 & 212501 & 37 & 38 & 37 & +1 & -23 & -9 \\
\hline 376 & 135634.9 & 232150 & 89 & 42 & 29 & +13 & -7 & -6 \\
\hline 377 & 143017.1 & 222555 & 127 & 30 & 22 & +8 & -7 & +1 \\
\hline
\end{tabular}


Hence, many ShCGs are associated with LGs, members of which, as in the case of brighter galaxies in the environments of some HCGs and poor galaxy groups with measured redshifts (Tovmassian \& Chavushyan 2000) or galaxies in the environment of HCGs with higher limiting magnitude $\left(20^{\mathrm{m}}\right)$ (Tovmassian 2001), are distributed along the elongation of the corresponding ShCGs. There are, on average, four galaxies in each LG. This shows that unavoidable selection effects in misclassification of the images of stars and galaxies in the APS do not have any significant influence on the statistical results, obtained by comparing the numbers of galaxies in orthogonal stripes, and do not mask the expected correlation.

Table 2. The mean values of differences $\Delta_{\mathrm{c}}, \Delta_{\mathrm{n}-\mathrm{s}}$, and $\Delta_{\mathrm{s}}$.

\begin{tabular}{rrr}
\hline$\Delta_{\mathrm{c}}$ & $\Delta_{\mathrm{n}-\mathrm{s}}$ & $\Delta_{\mathrm{s}}$ \\
\hline $3.7 \pm 6.9$ & $1.0 \pm 6.6$ & $-0.2 \pm 7.5$ \\
\hline
\end{tabular}

Redshifts of only 24 ShCGs out of 105 are known (Tiersch et al. 1996, 2001). Only one of these groups, ShCG 333, is at a redshift 0.011. Redshifts of other groups are in the range of $0.0517-0.185$. At a mean redshift of ShCGs, $z \sim 0.1$ (Tovmassian et al. 1998) the absolute magnitude of the faintest detected galaxy of the associated $\mathrm{LG}$ is, on average, equal to $-16^{\mathrm{m}}$. It means, that the members of the LGs associated with ShCGs are of moderate luminosities. The number of fainter galaxies could apparently be larger (Schechter 1976; Valotto et al. 1997).

It has been found that ShCGs have forms of prolate spheroids or "cigars" (Oleak et al. 1995, 1998). The distribution of the LG galaxies along the "cigar" shows that the whole $\mathrm{CG}+\mathrm{LG}$ system has the same shape. The peculiar distribution of $L G$ galaxies in narrow stripes in the environment of HCGs and of poor groups of galaxies, and the dependence of the RVD of LG members on the elongation of the whole system, allowed Tovmassian et al. (1998), Tovmassian \& Chavushyan (2000), and Tovmassian (2001) to conclude that the principal members of HCGs and the LG members associated with them move predominantly along the direction of elongation of the whole system. The situation is analogous in the case of ShCGs. Such space configuration and movement of members of ShCGs may be explained by three options:

1. Principal members and faint distant members of ShCGs depart from the center of group in opposite directions.

a. It could be a result of ejection from central galaxy in accordance with Ambartsumian's (1961) or Arp's (1973, 1997 and references therein) ideas. However, no physical mechanisms of such processes are yet known.

b. According to Binggeli (1982), clusters of galaxies with separations less than $30 h^{-1} \mathrm{Mpc}$ tend to "point towards each other". Splinter et al. (1997) showed by $N$-body simulations that alignments of cluster-cluster orientation drop off at distances $>15 h^{-1}$. Hence, a CG located between two nearby large clusters would disintegrate, and members of the former would depart from each other in the direction of the large cluster-cluster alignment. However, the distribution of orientation of major axes of HCGs is isotropic in relation to distribution of clusters of galaxies (Palumbo et al. 1995). Hence, there is no outside force to stretch the groups. The same could apparently be applied to ShCGs as well. Therefore, the first option should be ruled out.

2. Infall of environmental galaxies of filaments upon the group from opposite directions. The widths of filaments (see Figs. 5 and 6 in Eisenstein et al. 1997) are generally larger than the widths of the searched stripes $(\sim 600 \mathrm{kpc})$. It means that the infall should generally be isotropic, while the contrary is observed. Moreover, the orientation of the elongation of groups is not correlated with the distribution of nearby clusters (Palumbo et al. 1995). Therefore, this option should be ruled out as well.

3. The most realistic option which does not contradict to observational data is the following: principal members of the ShCG and faint members in its environment, distributed along the elongation of the group compose $a$ gravitationally bound system, and, probably, rotate around the gravitational center of the whole group in orbits preferentially oriented along the elongation of the group.

We may assume that a member of the CG makes one revolution around the gravitational center of the group during two crossing times, $2 \tau_{\mathrm{cr}}$. The half length of the elongated group is the semiaxis of the supposed orbit. The mean $\tau_{\text {cr }}$ of ShCGs is about $2 \times 10^{8}$ years, and the mean radius is about $120 \mathrm{kpc}$ (Tiersch et al. 2001). According to Kepler's third law, a LG galaxy at a mean distance of $0.5 \mathrm{Mpc}$ from the center of the CG makes one revolution in $\sim 5 \times 10^{9}$ years, i.e. in less than the Hubble time. The orbits of the principal members of the "cigar"-like systems may, however, be larger than the observed $\sim 100 \mathrm{kpc}$. For groups oriented close to the plane of the sky the observed length $r_{\mathrm{o}}=r_{\mathrm{t}} \cdot \cos \alpha$ would be near to the true value $r_{\mathrm{t}}$, while the observed RVD, $\sigma_{\mathrm{o}}=\sigma_{\mathrm{t}} \cdot \sin \alpha$ ( $\sigma_{\mathrm{t}}$ is the true value), would be smaller. For groups oriented near to the line of sight the observed length would be minimal, while the $\sigma_{\mathrm{o}}$ would be close to the real value. The revolution time of the LG member at a distance $a$ determined by the observed semiaxis $r_{\mathrm{o}}$ of the CG and its measured crossing time, $\tau_{\mathrm{cr}} \sim r_{\mathrm{o}} / \sigma_{\mathrm{o}}$, can be estimated:

$$
T \sim \sqrt{a^{3} / r_{\mathrm{o}} \sigma_{\mathrm{o}}^{2}}=\sqrt{a^{3}\left(r_{\mathrm{t}} \cdot \cos \alpha \cdot \sigma_{\mathrm{t}}^{2} \cdot(\sin \alpha)^{2}\right)^{-1}} .
$$

One has to take into account that $r_{\mathrm{o}}$ would never be zero, since galaxies in the group are not aligned along one line, and are moving in orbits with high excentricity. Similarly, $\sigma_{\mathrm{t}}$ would not be zero, since velocities of member galaxies in a group are not obviously parallel to each other. It follows then, that the deduced revolution time $T$ would be higher than the real value for groups oriented close to the line of sight, and also for those oriented nearly orthogonal 
to the line of sight. The estimated time would be nearer to the real value for groups with elongations oriented at intermediate angles to the line of sight. Therefore, the real rotation time would be smaller than the value estimated above by using the average values of the semiaxis and the crossing time of ShCGs. Hence, the CG+LG systems may well be virialized.

Near the gravitational center of a "cigar"-like system galaxies move faster. In such conditions efficiency of merging should apparently be small. However, in a "cigar"-like configuration with relatively small cross-section the effect, that sweeps out the gas by tidal forces from member galaxies passing-by not far from each other, may be very effective. This may partly explain the small spiral content of ShCGs, which is only $\sim 20 \%$ (Tiersch et al. 1996, 2001), meanwhile in HCGs, for example, the spirals compose about half of the population (Hickson et al. 1989). One has to take into account, however, that ShCGs were selected as groups of red galaxies. Therefore, the large content of ellipticals in them may be considered as a result of a bias. However, it is a fact that there exist such groups consisting mainly of ellipticals. It means that processes of interaction have, indeed, occurred in these groups, and they are not young formations, i.e. the ShCGs are dynamically more evolved than HCGs. It is natural that the rate of powerful infrared and radio sources in ShCGs, containing mostly gas poor ellipticals, is small (Tovmassian et al. 1998, 2000).

Hence, the option that members of LGs in the environment of ShCGs rotate around the common gravitational center preferentially in the direction of elongation of the CG, as was concluded for HCGs (Tovmassian \& Chavushyan 2000; Tovmassian 2001), seems to be acceptable in the case of ShCGs as well.

Thus, ShCGs seem to be more stable configurations than it has been supposed. The conclusion on very short life-times of CGs determined in $N$-body simulations, when only 5-10 galaxies have been considered, and when a probable regular rotational movement of member galaxies around the gravitational center of a group have been ignored (Barnes 1985, 1989; Bode et al. 1993; Mamon 1986), is hardly applicable to CGs generally. The ShCGs present an explicit observational evidence for it. The suggestion of Governato et al. (1991) and Zepf \& Whitmore (1991) on the quasi stationary orbits of most massive galaxies of CGs is nearer to reality.

\section{Conclusions}

The vicinities of 105 elongated ShCGs are searched for fainter members. Counts of galaxies are made in two orthogonal narrow stripes of $5^{\prime}$ width and $20^{\prime}$ length centered on each ShCG. The first stripe is oriented in the direction determined by the principal members of the corresponding group. Counts are made also in two half-as-narrow stripes on both sides of the central stripe.

About four galaxies are, on average, detected in the environment of each of the investigated 105 ShCGs.
In the case of 26 ShCGs with $\delta_{\mathrm{c}} \geq 8$ about 12 galaxies on average are found. The faint galaxies found are distributed along the direction of the elongation of the group. It is concluded that faint members together with the brighter members compose a gravitationally bound, most probably virialized system. The orbits of the member galaxies are preferetially aligned with the elongation of the group. Hence, ShCGs are more stable formations than has been assumed. Their new-found members of moderate luminosities are located at relatively large distances (up to $1 \mathrm{Mpc}$ ) from the visual central concentration of brighter galaxies. Apparently, ShCGs may contain fainter members. Therefore, these groups may be regarded as, rather, poor clusters of galaxies.

Processes of interaction, e.g. tidal stripping and ram pressure, are more likely in "cigar"-like ShCGs with small cross-sections than in ordinary poor groups or clusters of galaxies. At the same time, the processes of merging should be rarer for regular movement of member galaxies.

If the orientation of the elongated group is close to the line of sight, its bright members seen nearby on the sky may be far from each other in space. When the orientation of the group is nearly orthogonal to the line of sight, it would be detected as a CG only in the case when its bright members happen to be close to each other on the sky in the course of regular rotation around the gravitational center of the group. The probability of the latter is apparently very small. The groups without dominant bright members would also be undetected. It follows that the number of such groups in the nearby Universe could be much higher.

Acknowledgements. We are grateful to our anonymous referee for valuable comments by which the paper was significantly improved.

\section{References}

Ambartsumian, V. A. 1961, AJ, 66, 536

Arp, H. 1973, ApJ, 185, 797

Arp, H. 1997, ApJ, 474, 74

Baier, F. W., Petrosian, M. B., Tiersch, H., \& Shakhbazian, R. K. 1974, Astrofizika, 10, 327

Baier, F. W., \& Tiersch, H. 1975, Astrofizika, 11, 221

Baier, F. W., \& Tiersch, H. 1976a, Astrofizika, 12, 7

Baier, F. W., \& Tiersch, H. 1976b, Astrofizika, 12, 409

Baier, F. W., \& Tiersch, H. 1978, Astrofizika, 14, 229

Baier, F. W., \& Tiersch, H. 1979, Astrofizika, 15, 33

Barnes, J. E. 1985, MNRAS, 215, 517

Barnes, J. E. 1989, Nature, 338, 123

Barton, E. J., De Carvalho, R. R., \& Geller, M. J. 1998, AJ, 116,1573

Binggeli, B. 1982, A\&A, 107, 338

Bode, P. W., Cohn, H. N., \& Lugger, P. M. 1993, ApJ, 416, 17 Coziol, R., De Carvalho, R. R., Capelato, H. V., \& Ribeiro, A. L. B. 1998, ApJ, 506, 545

Diafero, A., Geller, M. J., \& Ramella, M. 1994, AJ, 107, 868

Diafero, A., Geller, M. J., \& Ramella, M. 1995, AJ, 109, 2293

Eisenstein, D. J., Loeb, A., \& Turner, E. L. 1997, ApJ, 475, 421

Governato, F., Bathia, R., \& Chincarini, G. 1991, ApJ, 371, L15 
Governato, F., Tozzy, P., \& Cavaliere, A. 1996, ApJ, 458, 18

Hernquist, L., Katz, N., \& Weinberg, D. H. 1995, ApJ, 442, 57

Hickson, P. 1997, ARA\&A, 35, 357

Hickson, P., \& Rood, H. J. 1988, ApJ, 331, L69

Hickson, P., Menon, T. K., Palumbo, G. G. C., \& Persic, M. 1989, ApJ, 341, 679

Mamon, G. A. 1986, ApJ, 307, 426

Mendes de Oliveira, C. 1995, MNRAS, 273, 139

Mendes de Oliveira, C., \& Giraud, E. 1994, ApJ, 437, L103

Oleak, H., Stoll, D., Tiersch, H., \& MacGillivray, H. T. 1995, AJ, 109, 1485

Oleak, H., Stoll, D., Tiersch, H., \& MacGillivray, H. T. 1998, Astron. Nachr., 319, 235

Ostriker, J. P., Lubin, L. M., \& Hernquist, L. 1995, ApJ, 444, L61

Palumbo, G. G. C., Saracco, P., Hickson, P., \& Mendes de Oliveira, C. 1995, AJ, 109, 1476

Petrosian, M. B. 1974, Astrofizika, 10, 471

Petrosian, M. B. 1978, Astrofizika, 10, 631

Ponman, T. J., Borner, P. D. J., Ebeling, H., \& Böhringer, H. 1996, MNRAS, 283, 690

Ramella, M., Diaferio, A., Geller, M. J., \& Huchra, J. P. 1994, AJ, 107, 1623

Ribeiro, A. L. B., De Carvalho, R. R., Capelato, H. V., \& Zepp, S. E. 1998, ApJ, 497, 72

Rood, H. J. 1979, ApJ, 233, 21

Rood, H. J., \& Williams, B. A. 1989, ApJ, 339, 772, RW

Rose, J. A. 1977, ApJ, 211, 311

Schechter, P. 1976, ApJ, 203, 297
Shakhbazian, R. K. 1973, Astrofizika, 9, 495

Shakhbazian, R. K., \& Petrosian, M. B. 1974, Astrofizika, 10, 13

Splinter, R. J., Melott, A. L., Linn, A. M., Buck, C., \& Tinker, J. 1997, ApJ, 479, 632

Sulentic, J. W. 1987, ApJ, 322, 605

Tiersch, H., Stoll, D., Neizvestny, S., Tovmassian, H. M., \& Navarro, S. 1996, J. Korean Astron. Soc., 29, 259

Tiersch, H., Stoll, D., Tovmassian, H. M., et al. 2001, in preparation

Tovmassian, H. M., Mazzarella, J. M., Tovmassian, G. H., Stoll, D., \& Tiersch, H. 1998, A\&AS, 130, 207

Tovmassian, H. M., Martinez, O., \& Tiersch, H. 1998, A\&A, 348,693

Tovmassian, H. M., Chavushyan, V. H., Verkhodanov, O. V., \& Tiersch, H. 1999, ApJ, 523, 1999

Tovmassian, H. M., \& Chavushyan, V. H. 2000, AJ, 119, 1687

Tovmassian, H. M., Mazzarella, J. M., Tovmassian, G. H., Stoll, D., \& Tiersch, H. 1998, A\&AS, 130, 207

Tovmassian, H. M. 2001, PASP, 113, 543

Tovmassian, H. M., Yam, O., \& Tiersch, H. 2001, Rev. Mex. A\&A, 37, October issue

Valotto, C. A., Nicotra, M. A., Muriel, H., \& Lamba, D. G. 1997, ApJ, 479, 90

Vennik, J., Richter, G. M., \& Longo, G. 1993, Astron. Nach., 314,393

Walke, D. G., \& Mamon, G. A. 1989, A\&A, 225, 291

Zepf, S. E., \& Whitmore, B. C. 1991, ApJ, 383, 524 